JOURNAL OF

SYNCHROTRON

RADIATION

ISSN 1600-5775

Received 15 June 2018

Accepted 23 November 2018

Edited by P. A. Pianetta, SLAC National Accelerator Laboratory, USA

Keywords: zone plate; diffraction limit; depth of focus; spatial resolution; microbeam.

Supporting information: this article has supporting information at journals.iucr.org/s

\section{Inverse-phase composite zone plate providing deeper focus than the normal diffraction-limited depth of X-ray microbeams}

\author{
Yasushi Kagoshima* and Yuki Takayama \\ Graduate School of Material Science, University of Hyogo, 3-2-1 Kouto, Kamigori, Ako, Hyogo 678-1297, Japan. \\ *Correspondence e-mail: kagosima@sci.u-hyogo.ac.jp
}

A novel type of zone plate $(\mathrm{ZP})$, termed an inverse-phase composite $\mathrm{ZP}$, is proposed to gain a deeper focus than the standard diffraction-limited depth of focus, with little reduction in spatial resolution. The structure is a combination of an inner $\mathrm{ZP}$ functioning as a conventional phase $\mathrm{ZP}$ and an outer $\mathrm{ZP}$ functioning with third-order diffraction with opposite phase to the inner ZP. Twodimensional complex amplitude distributions neighboring the focal point were calculated using a wave-optical approach of diffraction integration with a monochromatic plane-wave illumination, where one dimension is the radial direction and the other dimension is the optical-axis direction. The depth of focus and the spatial resolution were examined as the main focusing properties. Two characteristic promising cases regarding the depth of focus were found: a pit-intensity focus with the deepest depth of focus, and a flat-intensity focus with deeper depth of focus than usual ZPs. It was found that twice the depth of focus could be expected with little reduction in the spatial resolution for $10 \mathrm{keV}$ X-ray energy, tantalum zone material, $84 \mathrm{~nm}$ minimum fabrication zone width, and zone thickness of $2.645 \mu \mathrm{m}$. It was also found that the depth of focus and the spatial resolution were almost unchanged in the photon energy range from 8 to $12 \mathrm{keV}$. The inverse-phase composite ZP has high potential for use in analysis of practical thick samples in X-ray microbeam applications.

\section{Introduction}

Fresnel zone plates (ZPs) are major optical elements in X-ray microscopes. There are several derivations such as sputteredsliced ZPs (Rudolph et al., 1982; Koyama et al., 2012), multilayer Laue lenses (Maser et al., 2004; Koyama et al., 2008) and total reflection ZPs (Takano et al., 2010). There are other types of optical elements such as grazing-incidence total reflection mirrors of Kirkpatrick-Baez mirrors (Mimura et al., 2007) and Wolter type I mirrors (Aoki et al., 1992), compound refractive lenses (Schroer et al., 2005) and Bragg-Fresnel lenses (Erko et al., 1994). The development of these optical elements has made sub-100 nm-spatial resolution easily available in X-ray microscopes. As the spatial resolution becomes higher, the demand for localized analysis of practical samples is rapidly increasing. However, according to the physical principle of the diffraction limit, the higher the spatial resolution (the smaller the focused beam size), the shallower the depth of focus in a focusing optical system. Optical elements have to date been unable to evade the diffraction limit. The diffraction limit in optics, which corresponds to the uncertainty principle in quantum mechanics, is usually expressed as being that the product of the spatial resolution $\left(\Delta_{\text {res }}\right)$ and the numerical aperture (NA) cannot be smaller than the wavelength, namely, $\Delta_{\text {res }} \times \mathrm{NA} \geq \lambda$. Because the depth of focus $(\mathrm{DoF})$ is also 
determined by NA such that DoF $\propto 1 / \mathrm{NA}^{2}$, improved spatial resolution and deeper DoF are incompatible. This can be interpreted as an alternative expression of the diffraction limit. This limit restricts the thickness of samples to be analyzed and is an impediment to high-spatial-resolution micro-analysis of practical samples using X-ray microscopes. In particular, the DoF is a critical parameter for X-ray microCT (computed tomography) with X-ray microscopes. The DoF of a ZP was well studied both with simulations and experiments aiming at high-spatial-resolution $\mathrm{X}$-ray micro-CT (Wang et al., 2000).

A novel type of $\mathrm{ZP}$ has been proposed, named an inversephase composite ZP (IP-CZP), in order to relax this limit, with an initial solution that facilitates a deeper DoF with little reduction of the spatial resolution (Kagoshima \& Takayama, 2018). This paper reports on the focusing properties of the IP-CZP from the point of view of both DoF and spatial resolution.

It should be noted here that another way to produce a blurred focal spot with an extended DoF has been recently reported using multiple zone plate stacking with misalignment along the optical axis (Li \& Jacobsen, 2018).

\section{Inverse-phase composite ZP}

\subsection{Principle and strategy to make DoF deeper}

There are two types of ZPs, positive and negative, as shown in Figs. 1(a) and 1(b). Both ZPs function as thin lenses. They have identical focal intensity distributions in the focal plane and along the optical axis, that determine $\Delta_{\text {res }}$ and DoF, respectively. On the other hand, the phase of the complex amplitude of focusing waves is opposite to each other. A composite ZP was proposed, and its optical properties were well studied (Michette, 1986). If the inner ZP with a first-order focal length of $f$ is surrounded by the outer ZP with a thirdorder focal length also of $f$, the effective NA becomes larger as shown in Fig. 1(c). This leads to higher spatial resolution (smaller focused beam size) and an increase of the focused intensity.

In order to deepen the DoF without reducing the spatial resolution, the focal intensity distribution along the optical axis should be broadened, whereas the focusing beam size in the focal plane should be as unchanged as possible. Our strategy to accomplish this is as follows. We previously proposed that the phase of the inner ZP (iZP) and outer ZP (oZP) are inversely composed as shown in Fig. 1(d) (Kagoshima \& Takayama, 2018). Because the aperture of the oZP is annular, the focused beam size produced only by the oZP becomes narrower than that of a circular aperture with the same NA. As the effective number of zones of the oZP becomes smaller, the monochromaticity require-

Figure 2 (a)

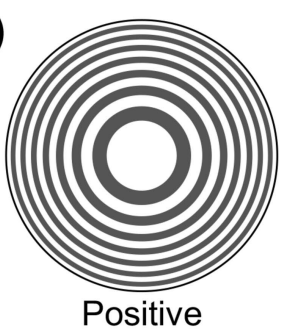

(c)

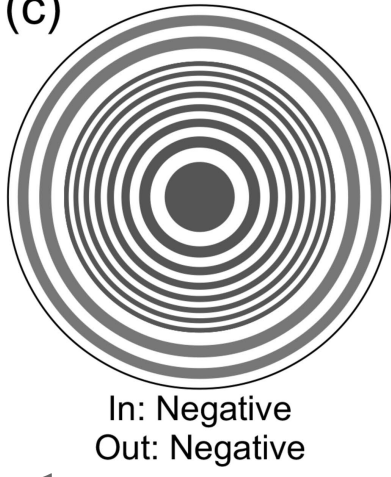

(b)

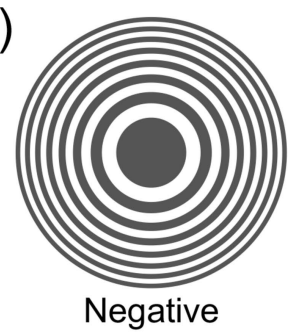

(d)

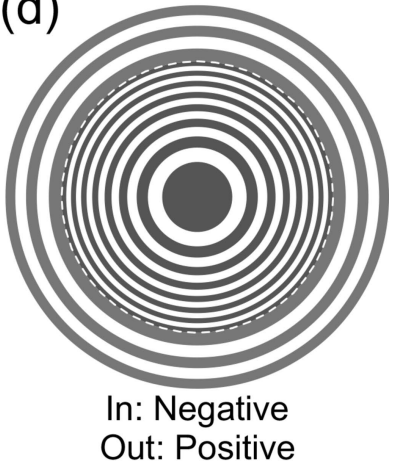

Out: Positive

Figure 1

(a) Positive ZP, (b) negative ZP, (c) composite ZP and (d) inverse-phase composite ZP (IP-CZP). The gray color of the zones indicates that ZPs function as a phase zone plate. The white dotted circle in $(d)$ is the boundary of the iZP and oZP.

ment for the oZP becomes looser, which leads to the broadening in the focal intensity distribution of the oZP along the optical axis. The diffraction efficiency of ZPs depends on the zones' material and thickness. If opaque zones act as phase material, ZPs work as phase ZPs (Kirz, 1974). By tuning the thicknesses of the iZP and oZP independently, the DoF and $\Delta_{\text {res }}$ of the IP-CZP can be controlled because the complex amplitude of the IP-CZP is the coherent sum of each complex amplitude of the iZP and oZP. Therefore, if the parameters of the IP-CZP are appropriately chosen, the DoF could be deepened with little reduction of the spatial resolution.

\subsection{Definitions of design parameters}

Definition of the main variable design parameters of the IPCZP are illustrated in Fig. 2. Those of the iZP are the first zone radius, $r_{1 \_ \text {in }}$, outer radius, $r_{N_{-} \text {in }}$, outermost zone width, $\Delta r_{N_{-} \text {in }}$,

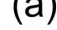

(b)

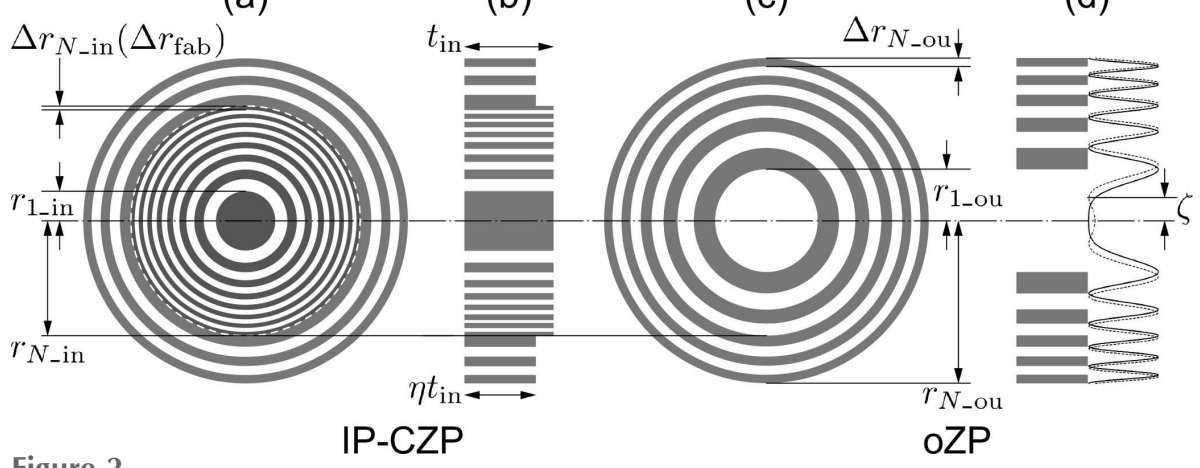

Definition of the main design parameters of the IP-CZP. (a) IP-CZP front view and $(b)$ section; (c) outer ZP front view and $(d)$ section. 
which is supposed to be equal to the minimum fabrication width $\Delta r_{\text {fab }}$, and zone thickness, $t_{\text {in }}$. Those of the oZP are the first zone radius, $r_{1 \_ \text {ou }}$, outer radius, $r_{N_{-} \text {ou }}$, outermost zone width, $\Delta r_{N_{-} \text {ou }}$, and zone thickness, $\eta t_{\text {in }}$, where $\eta$ is the thickness ratio. In this paper, it is assumed that $\eta \leq 1$. $r_{N_{-} \text {ou }}$ is identical to an outer radius of IP-CZP and represented by an annular parameter $\varepsilon$ as $r_{N_{-} \text {ou }}=r_{N_{-} \text {in }} / \varepsilon$. The focal length of the firstorder diffraction of iZP, $f_{1 \_ \text {in }}$, is given by $r_{1 \_ \text {in }}^{2} / \lambda$. Since the focal length of the third-order diffraction of the oZP, $f_{3 \_ \text {ou }}$, is equal to $f_{1 \_ \text {in }}, r_{1 \_ \text {ou }}$ is $\sqrt{3} r_{1 \_ \text {in }}$. The local diffraction efficiency is determined by the refractive index of the zone material, $n=$ $1-\delta+i \beta$, and the zone thickness (Kirz, 1974). The initial phase of oZP, $\zeta$, should also be able to be tuned for the optimization. When $\zeta=\pi$ and $\eta=1$, the IP-CZP is identical to a CZP. The effective number of zones of the oZP, $N_{\text {ou_eff }}$, is $N_{\text {ou_eff }}=N_{\text {ou }}-N_{\text {in }} / 3$.

\subsection{Spatial resolution $\left(\Delta_{\text {res }}\right)$ and DoF}

The two main focusing properties of optical elements are the spatial resolution $\left(\Delta_{\text {res }}\right)$ and the DoF. The former can be evaluated from the radial intensity distribution in the focal plane perpendicular to the optical axis. The latter can be evaluated from the intensity distribution along the optical axis neighboring the focal point. Both are defined by the numerical aperture (NA) as follows,

$$
\begin{aligned}
\mathrm{NA} & =m \sin \theta, \\
\Delta_{\text {res }} & =\frac{0.610 \lambda}{\mathrm{NA}}, \\
\mathrm{DoF} & = \pm \frac{1}{2} \frac{\lambda}{\mathrm{NA}^{2}},
\end{aligned}
$$

where $m$ is the refractive index of the object space and $\theta$ is the objective angular semi-aperture. Equation (2) is well known as the Rayleigh criterion and usually understood to express the diffraction limit in microscopes. $\Delta_{\text {res }}$ is the same as the radius of the first null of the Airy pattern. DoF corresponds to a range within which the on-optical-axis intensity decreases by $20 \%$ from the peak intensity (Born \& Wolf, 1986). Therefore, the smaller the $\Delta_{\text {res }}$, the shallower the DoF as given by

$$
\mathrm{DoF}= \pm 1.34 \frac{\Delta_{\text {res }}^{2}}{\lambda} \text {. }
$$

As described above, equation (4) is another expression of the diffraction limit for a circular aperture.

For a usual ZP, $\Delta_{\text {res }}$ and DoF can be given by the outermost (narrowest fabricated) zone width, $\Delta r_{N}$, as follows (Attwood, 1999),

$$
\begin{aligned}
& \Delta_{\text {res }}=1.22 \Delta r_{N}, \\
& \mathrm{DoF}= \pm 2 \frac{\Delta r_{N}^{2}}{\lambda} .
\end{aligned}
$$

The achievable $\Delta_{\text {res }}$ depends only on $\Delta r_{N}$, whereas DoF depends both on $\Delta r_{N}$ and $\lambda$. The monochromaticity required to achieve equation (5) is $\lambda / \Delta \lambda \geq N$, where $N$ is the total number of zones. If this condition is not satisfied, both $\Delta_{\text {res }}$ and DoF become larger than equations (5) and (6) corresponding to the degree of monochromaticity. In this paper, hereafter, $\Delta_{\text {res }}$ is defined as a radius initially yielding effective null in the intensity distribution.

\subsection{Relevant design parameters}

High-energy X-rays enable experiments to be conducted under atmospheric conditions, which is advantageous for the structural analysis of practical samples. Considering this, the operation X-ray energy of the IP-CZP is at the relatively high energy of $10 \mathrm{keV}$. The target $\Delta_{\text {res }}$ is set to be about $100 \mathrm{~nm}$. Since the monochromaticity, $\lambda / \Delta \lambda$, of a silicon (111) doublecrystal monochromator typically used in synchrotron radiation beamlines is of the order of several thousands, the effective total number of zones, $N_{\text {tot_eff }}=3 N_{\text {ou }}$, must be smaller than $\lambda / \Delta \lambda$. The focal length should not be too small in order to maintain a practical working distance.

In order to be consistent with our previous studies (Ozawa et al., 1997), tantalum is chosen as the zone material. According to the data tables (Henke et al., 1993; Center for X-ray Optics, http://henke.lbl.gov/optical_constants/tgrat2. html), the refractive index of tantalum at $10 \mathrm{keV}$ is $\delta=$ $2.34356557 \times 10^{-5}$ and $\beta=3.89601519 \times 10^{-6}$. As an initial design step, the zone thickness of the iZP, $t_{\text {in }}$, is chosen to be $\lambda / 2 \delta=2.645 \mu \mathrm{m}$, yielding a phase shift of $\pi$, which means that the iZP works as an ordinary phase $\mathrm{ZP}$ when neglecting absorption.

Considering the above boundary conditions, $r_{1 \_ \text {in }}$ and $r_{1 \_ \text {ou }}$ are set to be $2.50 \mu \mathrm{m}$ and $4.33 \mu \mathrm{m}$, respectively, which gives a focal length $f_{1 \_ \text {in }}$ of $50.41 \mathrm{~mm}$ at $10 \mathrm{keV} . \Delta r_{N_{-} \text {in }}\left(=\Delta r_{\text {fab }}\right)$ is set to be $84 \mathrm{~nm}$. Thus, $r_{N \_ \text {in }}$ becomes $37.25 \mu \mathrm{m}$ with $N_{\text {in }}=222$. By modifying $N_{\text {ou }}$ (equally $\varepsilon$ ), $\eta$ and $\zeta$, the performance of $\Delta_{\text {res }}$ and DoF has been optimized.

\section{Calculation of optical properties}

\subsection{Diffraction integration}

The three-dimensional complex amplitude distribution neighboring the focal point, $A(r, \theta, z)$, has been calculated according to the diffraction integration with a monochromatic plane-wave illumination of wavelength $\lambda$ propagating along the $z$-axis. The optical system is shown in Fig. 3. If those of the iZP and oZP are denoted independently as $A_{\text {in }}(r, \theta, z)$ and $A_{\text {ou }}(r, \theta, z)$, respectively, the diffraction integration is written as follows in cylindrical coordinates,

$$
A(r, \theta, z)=A_{\text {in }}(r, \theta, z)+A_{\text {ou }}(r, \theta, z)
$$

$$
A_{\text {in }}(r, \theta, z) \propto \frac{1}{\lambda} \int_{0}^{2 \pi} \int_{0}^{r_{N-\text { in }}} \exp \left[i k n_{\text {in }}(\rho, \varphi) t_{\text {in }}\right] \frac{\exp (i k l)}{l} \rho \mathrm{d} \rho \mathrm{d} \varphi,
$$


Table 1

Parameters of two promising IP-CZPs. ZP- $A$ has a pit-intensity focus with the deepest DoF and ZP- $B$ has a flat-intensity focus with deeper DoF than usual ZPs.

\begin{tabular}{|c|c|c|c|c|c|c|c|c|c|c|c|c|c|}
\hline $\mathrm{ZP}$ & $\begin{array}{l}r_{1 \_ \text {in }} \\
(\mu \mathrm{m})\end{array}$ & $\begin{array}{l}r_{N \_ \text {in }} \\
(\mu \mathrm{m})\end{array}$ & $N_{\text {in }}$ & $\begin{array}{l}\Delta r_{N_{\text {in }}} \\
(\mathrm{nm})\end{array}$ & $\begin{array}{l}r_{1 \_ \text {ou }} \\
(\mu \mathrm{m})\end{array}$ & $\begin{array}{l}r_{N \_ \text {ou }} \\
(\mu \mathrm{m})\end{array}$ & $N_{\text {ou }}$ & $N_{\text {ou_eff }}$ & $\begin{array}{l}\Delta r_{N_{-} \mathrm{ou}} \\
(\mathrm{nm})\end{array}$ & $N_{\text {tot_eff }}$ & $\varepsilon$ & $\eta$ & $\zeta$ \\
\hline $\begin{array}{l}A \\
B\end{array}$ & 2.50 & 37.25 & 222 & 84 & 4.33 & $\begin{array}{l}47.63 \\
44.58\end{array}$ & $\begin{array}{l}121 \\
106\end{array}$ & $\begin{array}{l}47 \\
32\end{array}$ & $\begin{array}{l}197 \\
211\end{array}$ & $\begin{array}{l}363 \\
318\end{array}$ & $\begin{array}{l}0.782 \\
0.836\end{array}$ & $\begin{array}{l}1.00 \\
0.85\end{array}$ & $\begin{array}{l}0 \\
0\end{array}$ \\
\hline
\end{tabular}

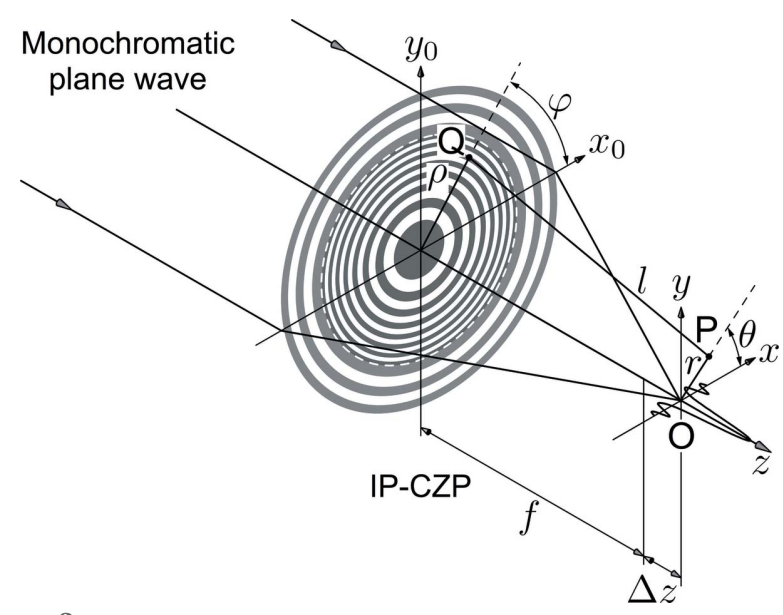

Figure 3

Optical system for diffraction integration with a monochromatic planewave illumination. $(\rho, \varphi)$ and $\left(x_{0}, y_{0}\right)$ are coordinates in the IP-CZP plane, and $(r, \theta)$ and $(x, y)$ are those in the observation plane at a distance $z$ $(=f+\Delta z)$ from the IP-CZP. $l$ is the distance from a point $Q$ in the IP-CZP plane to a point $P$ in the observation plane.

$$
A_{\text {ou }}(r, \theta, z) \propto \frac{1}{\lambda} \int_{0}^{2 \pi} \int_{r_{N_{-} \text {in }}}^{r_{N}-\text { ou }} \exp \left[i k n_{\text {ou }}(\rho, \varphi) t_{\text {in }}\right] \frac{\exp (i k l)}{l} \rho \mathrm{d} \rho \mathrm{d} \varphi,
$$

$$
\begin{gathered}
I(r, \theta, z)=|A(r, \theta, z)|^{2}, \\
l=\left[\left(x-x_{0}\right)^{2}+\left(y-y_{0}\right)^{2}+z^{2}\right]^{1 / 2}, \quad z=f+\Delta z, \\
x_{0}=\rho \cos \varphi, \quad y_{0}=\rho \sin \varphi, \quad x=r \cos \theta, \quad y=r \sin \theta
\end{gathered}
$$

In the above, $(\rho, \varphi)$ and $\left(x_{0}, y_{0}\right)$ are polar and Cartesian coordinates in the IP-CZP plane, and $(r, \theta)$ and $(x, y)$ are those in the observation plane at a distance $z(=f+\Delta z)$ from the IPCZ. $l$ is a distance from a point $Q$ in the IP-CZP plane to a point $P$ in the observation plane. $n_{\text {in }}(\rho, \varphi)$ and $n_{\text {ou }}(\rho, \varphi)$ are the refractive index distributions of the $\mathrm{iZP}$ and $\mathrm{oZP}$, respectively. $I(r, \theta, z)$ is the intensity. In the case when $\eta \neq 1$ for the oZP, $n_{\text {ou }}$ in the thickness direction between $\eta t_{\text {in }}$ and $t_{\text {in }}$, shown in Fig. 2(b), is replaced by that of the surrounding environment. This scalar wave simulation is valid for the evaluation of the proposed ICP-ZPs as discussed in Section S1 of the supporting information.

\subsection{Two promising cases}

Diffraction integration has been performed to calculate the two-dimensional complex amplitude distribution (magnitude, phase, real and imaginary parts) neighboring the focal point. One dimension is the radial direction, $r$, and the other dimension is the optical axis direction, $\Delta z$. The intensity is the sum of the squares of the real and imaginary parts.

Two characteristic promising cases were found: a pitintensity focus with the deepest $\operatorname{DoF}(\mathrm{ZP}-A)$ and a flatintensity focus with deeper $\operatorname{DoF}(\mathrm{ZP}-B)$ than a usual ZP. Table 1 shows parameters of the two promising cases. The iZPs are identical between the two. The diameter of the oZP of ZP$A$ is larger than that of $\mathrm{ZP}-B$ with $\varepsilon$ of 0.782 and 0.836 , respectively. The thickness of the oZP of $\mathrm{ZP}-A$ is larger than that of ZP- $B$ with $\eta$ of 1.00 and 0.85 , respectively. The outermost zone width of the two oZPs, $\Delta r_{N_{-} \text {ou }}$, is larger than twice that of the iZP, $\Delta r_{N_{-} \text {in }}$, of $84 \mathrm{~nm}$. The effective total number of zones, $N_{\text {tot_eff }}$, is sufficiently smaller than several thousands, which means that the chromatic aberration is negligible in usual synchrotron radiation beamlines equipping standard silicon double-crystal monochromators.

Fig. 4 shows the calculated two-dimensional intensity distribution, $I(r, \Delta z)$, of (a) ZP- $A$, (b) ZP- $B$ and (c) iZP-only. The intensity is a normalized value by the peak intensity of iZP-only. The black dashed lines denote contours of $80 \%$ intensity relative to the self-peak intensity to make regions of each DoF clear. The ZP- $A$ exhibits features of a pit-intensity focus and $\mathrm{ZP}-B$ exhibits features of a flat-intensity focus along the $\Delta z$ axis. Fig. 5 shows intensity distributions $(a)$ in the radial-direction, $r$, at $\Delta z=0$ and (b) along the optical-axis, $\Delta z$, at $r=0$ of ZP- $A$ and ZP- $B$ accompanied by those of iZP-only. The former corresponds to the point spread functions (PSFs) and the latter determines DoF. The PSF of iZP-only [black in Fig. $5(a)]$ is in accord with the theoretical values of $\left[2 J_{1}(x) / x\right]^{2}$, where $x=(2 \pi / \lambda) r_{N \_ \text {in }} r / z$ (Born \& Wolf, 1986). The intensity distribution along the optical axis of iZP-only [black in Fig. 5(b)] is also in accord with the theoretical values of $[\sin (u / 4) /(u / 4)]^{2}$, where $u / 4=\pi r_{N \text { in }}^{2} \Delta z / 2 \lambda z^{2}$ (Born \& Wolf, 1986).

In this paper, $\Delta_{\text {res }}$ is defined as a radius initially yielding effective null in the intensity distribution. Each $\Delta_{\text {res }}$ is shown in Fig. 5(a). According to equation (4), the increase of DoF can be assessed. The calculation results relating to $\Delta_{\text {res }}$ and DoF are summarized in Table 2. The calculation results relating to intensity are summarized in Table $3 . \Delta_{\text {res }}$ is $110 \mathrm{~nm}$ and $109 \mathrm{~nm}$, and DoF is $461 \mu \mathrm{m}$ and $412 \mu \mathrm{m}$ for $\mathrm{ZP}-A$ and ZP$B$, respectively. Because $\Delta_{\text {res }}$ determined by $\Delta r_{\text {fab }}$ is $102 \mathrm{~nm}$ 


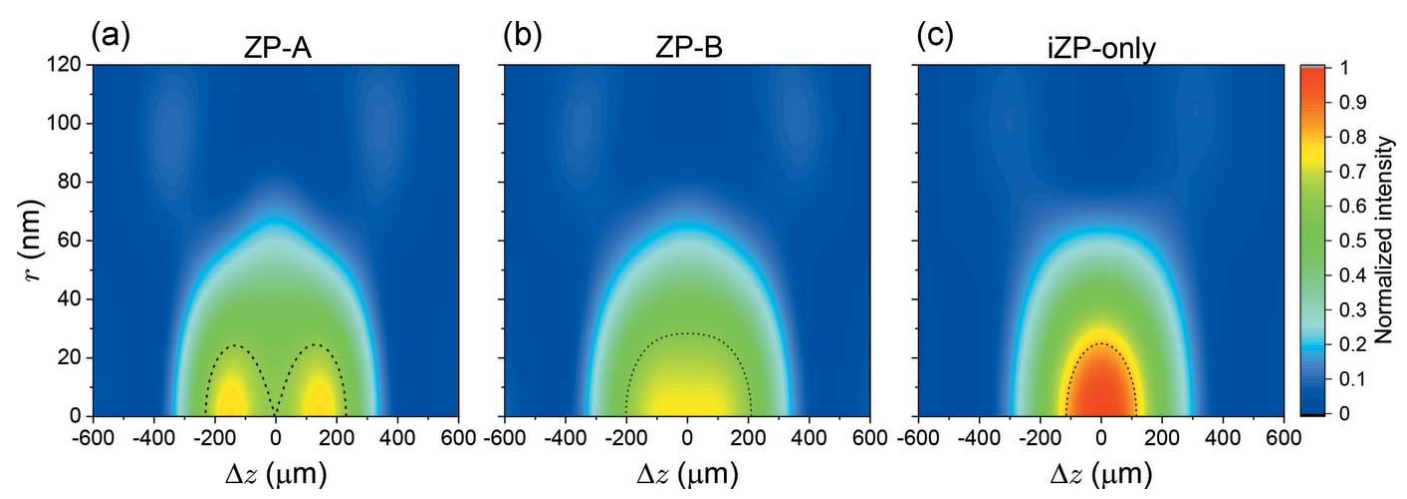

Figure 4

Calculated two-dimensional intensity distributions: $(a) \mathrm{ZP}-A,(b) \mathrm{ZP}-B$ and $(c)$ iZP-only. The intensity is a normalized value by the peak intensity of iZPonly. The black dashed lines denote contours of $80 \%$ intensity relative to self-peak intensity.

Table 2

Summary of the calculation results relating to $\Delta_{\text {res }}$ and DoF.

\begin{tabular}{llllllll}
\hline ZP & $\begin{array}{l}\Delta_{\text {res }} \\
(\mathrm{nm})\end{array}$ & $\begin{array}{l}\text { FWHM } \\
(\mathrm{nm})\end{array}$ & $\begin{array}{l}\text { DoF } \\
(\mu \mathrm{m})\end{array}$ & $\begin{array}{l}\text { DoF } \\
\text { Eq. }(4) \\
(\mu \mathrm{m})\end{array}$ & $\begin{array}{l}\text { DoF/ } \\
\operatorname{DoF}(\mathrm{iZP})\end{array}$ & $\begin{array}{l}1.22 \Delta r_{\text {fab }} \\
(\mathrm{nm})\end{array}$ & $\begin{array}{l}\Delta_{\text {res }} / \\
\Delta_{\text {res }}(\mathrm{iZP})\end{array}$ \\
\hline$A$ & 110 & 109 & 461 & 262 & 2.00 & 102 & 1.08 \\
$B$ & 109 & 98 & 412 & 258 & 1.79 & 102 & 1.07 \\
iZP-only & 102 & 86 & 231 & 226 & 1 & 102 & 1 \\
\hline
\end{tabular}

(a)

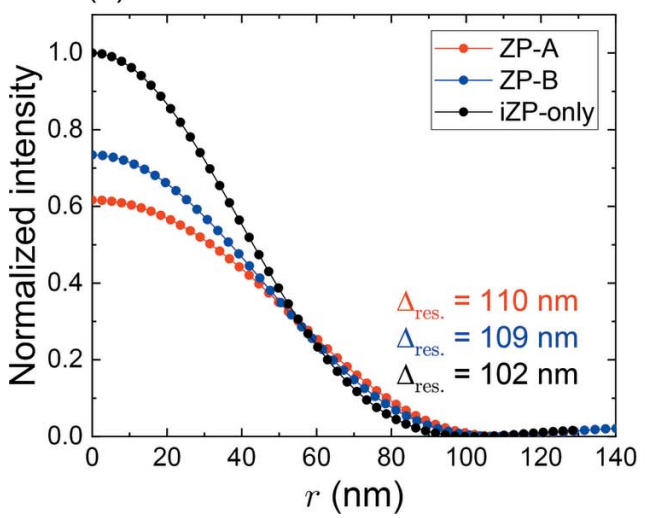

Figure 5

(a) Calculated point spread functions at $\Delta z=0$ and $(b)$ intensity distributions along the optical axis, $\Delta z$, at $r=0$. The ordinate is a normalized value by the peak intensity of iZP-only. $\Delta_{\text {res }}$ of ZP- $A, \mathrm{ZP}-$ $B$ and iZP-only are $110 \mathrm{~nm}, 109 \mathrm{~nm}$ and $102 \mathrm{~nm}$, respectively. DoFs of ZP- $A, \mathrm{ZP}-B$ and iZP-only are $461 \mu \mathrm{m}, 412 \mu \mathrm{m}$ and $231 \mu \mathrm{m}$, respectively.

Table 3

Summary of the calculation results relating to intensity.

Values are relative values to those of iZP-only.

\begin{tabular}{llll}
\hline $\mathrm{ZP}$ & $I_{\max }$ & $I_{\text {int }}$ & Normalized $I_{\text {int }}$ \\
\hline$A$ & 0.77 & 0.77 & 0.47 \\
$B$ & 0.73 & 0.75 & 0.52 \\
iZP-only & 1 & 1 & 1 \\
\hline
\end{tabular}

$\left(=1.22 \Delta r_{\text {fab }}\right)$, the reduction in the spatial resolution is as small as $8 \%$ for both ZPs, from the point of view of fabrication. On the other hand, DoF can be 2.0 and 1.8 times deeper than that of iZP-only. The maximum intensity is reduced to $77 \%$ and $73 \%$ for $\mathrm{ZP}-A$ and $\mathrm{ZP}-B$, respectively, in comparison with that of iZP-only. The integrated intensity, $I_{\text {int }}$, within which the sum of intensities decreases by $20 \%$ from the self-peak intensity (inside the black dotted lines in Fig. 4), is reduced to $77 \%$ and $75 \%$ for $\mathrm{ZP}-A$ and $\mathrm{ZP}-B$, respectively, in comparison with that of iZP-only. We found that a DoF of twice the depth could be expected with little reduction in the spatial resolution and with $\sim 1 / 4$ intensity reduction.

It should be mentioned here that the focused intensity of $\mathrm{ZP}-A$ and $\mathrm{ZP}-B$ normalized by the total incident $\mathrm{X}$-ray intensity, namely efficiency, is low due to both intentionally adapting the inverse phase of oZP and to larger diameters than that of iZP-only. The normalized integrated intensity by the area of the ZPs, which corresponds to efficiency, is also shown in Table 3 . The efficiency of $\mathrm{ZP}-A$ and $\mathrm{ZP}-B$ is about half that of iZP-only. In synchrotron radiation beamlines, the incident beam size at the experimental station is usually larger than the size of practical ZPs. The larger incident beam size is also required to allow for the beam drift. Thus, the actual focused intensity may be more practically important than the efficiency.

\subsection{Dependence on photon energy}

The focusing property dependence on photon energy has also been investigated. Fig. 6 shows PSFs of $(a) \mathrm{ZP}-A$ and $(b)$ $\mathrm{ZP}-B$ for several photon energies from 8 to $12 \mathrm{keV}$, respectively. All PSFs self-normalized by the intensity at $r=0$ have almost the same profile (not shown in the figure). Thus, the dependence of $\Delta_{\text {res }}$ on wavelength is negligible in the photon energy range. On the other hand, regarding intensity distributions along the optical axis, $\Delta z$, at $r=0$, the situation is somewhat different as shown in Fig. 7, where the abscissa, $\Delta z$, 
(a)

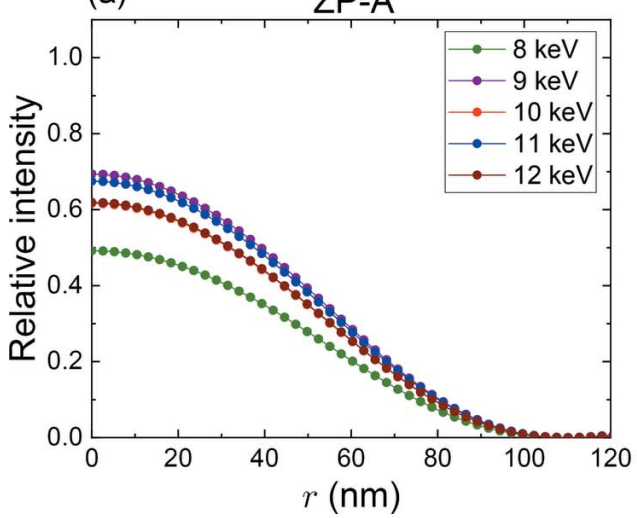

(b)

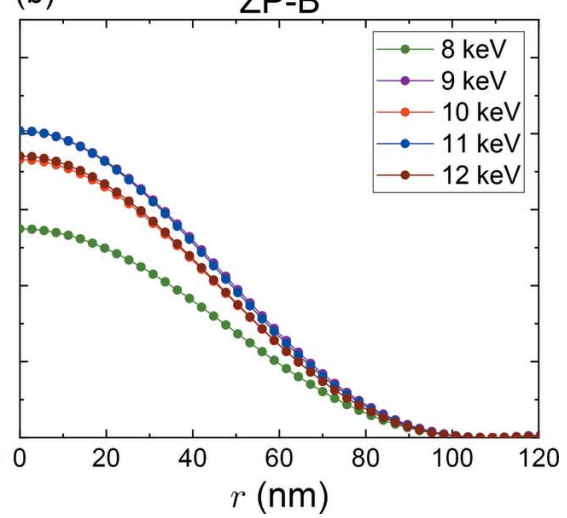

Figure 6

Calculated point spread functions at $\Delta z=0$ at several photon energies: (a) ZP- $A$ and (b) ZP- $B$. The ordinate is a relative value to the peak intensity of iZP-only at the photon energy of $10 \mathrm{keV}$.

energy range. These respective properties may not be simply understood, and further explanation is outside the scope of this paper. Fig. 8 shows $(a)$ intensity changes at $\Delta z=0$ and $r=0$, and $(b)$ DoF and DoF $/ f$ changes. The diffraction efficiency of a phase zone plate depends on both the real part $\delta$ and the imaginary part $\beta$ of the refractive index of the zone material (Kirz, 1974). The same theory can be applied to the present IP-CZP as a linear combination of complex amplitude of the iZP and oZP. The $L$-absorption edges of the zone material (tantalum) are indicated as a reference by vertical black arrows. The absorption edges have no influence is a distance from a focus position of each photon energy. ZP$A$ almost conserves the line symmetry about the line of $\Delta z=0$, while the line symmetry breaks for ZP- $B$ in the lower photon energies. It is fortunate for $\mathrm{ZP}-A$ that the normalized intensity of the pits $(\Delta z=0)$ by the self-maximum intensity is constantly 0.8 , and thus the deepest DoF can be maintained in the photon

on DoF. DoF increases linearly, while DoF/ $f$ is almost constant because $f$ is proportional to the photon energy.

\section{Future prospects}

Difficulties in the fabrication are not considered in this paper. Actually, the aspect ratio of the IP-CZP presented in this paper is 31.5 , which is too high to realize by the presently popular fabrication technique of electron beam lithography. Solutions for fabricating high-aspect-ratio ZPs were demonstrated by stacking two identical zone plates (Snigireva et al., 2007; Kagoshima et al., 2011), double-sided zone plates (Mohacsi et al., 2017) and zone plates of the highest aspect ratio fabricated by using metal-assisted chemical etching ( $\mathrm{Li}$ et al., 2017). Progress on these advanced fabrication technologies will make the proposed IPCZPs available.

Another surpassing way to produce a blurred focal spot with an extended DoF has been studied (Li \& Jacobsen, 2018). It uses multiple zone plate stacking with misalignment along the optical axis. The merit of producing blurred or extended DoF by multiple zone plate stacking is higher efficiency, i.e. zone plate stacking brings more energy to the focus. On the other hand, our IP-CZP has the great advantage that it is a single lens on a single substrate, and thus it can be easily handled and operated as a thin lens though the efficiency is low. Further, the focused intensity can be compensated by using brighter sources. Since $\Delta_{\text {res }}$ and DoF are naturally determined by the

Figure 8

(a) Calculated intensity changes at $\Delta z=0$ and $r=0$, and (b) DoF (left) and DoF/f (right) at several photon energies. The ordinate of $(a)$ is a relative value to the peak intensity of iZP-only at the photon energy of $10 \mathrm{keV}$. In $(b)$, the solid and dotted lines correspond to DoF and DoF/f, respectively. $L$-absorption edges of tantalum are indicated.

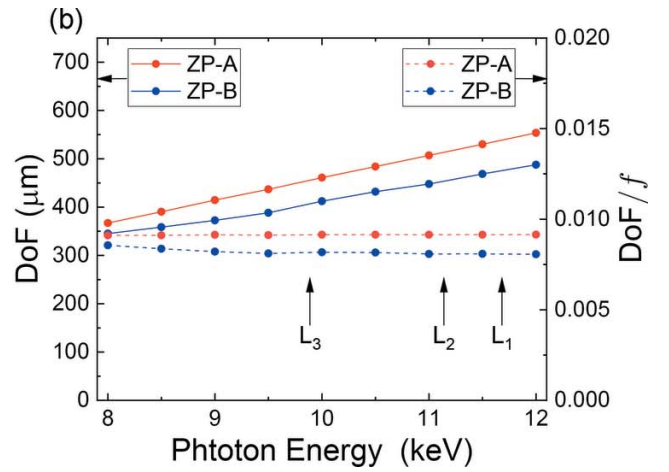

J. Synchrotron Rad. (2019). 26, 52-58

Kagoshima and Takayama • Inverse-phase composite zone plate 57 
numerical aperture of an optical device itself, they are unable to be gained by the source intensity. Therefore, the IP-CZP will be practically valuable for thicker samples especially with future brighter sources.

\section{Conclusions}

In order to defeat the diffraction limit restricting the relation between spatial resolution and depth of focus, an inversephase composite zone plate is proposed. The structure is a combination of an iZP functioning as a conventional phase zone plate and an oZP functioning with third-order diffraction with opposite phase to the iZP. The focusing properties of spatial resolution and DoF at a photon energy of $10 \mathrm{keV}$ have been investigated by diffraction integration. Two characteristic promising cases were found of a pit-intensity focus with the deepest DoF and a flat-intensity focus with deeper DoF than usual ZPs. We found that a DoF of nearly twice the depth could be expected with little reduction in the spatial resolution, and that DoF and the spatial resolution were almost unchanged in the relatively wide photon energy range.

\section{Related literature}

The following references, not cited in the main body of the paper, have been cited in the supporting information: Kang et al. (2005, 2006); Maser \& Schmahl (1992); Schneider (1997); Schnopper et al. (1977).

\section{Acknowledgements}

We would like to thank Editage (https://www.editage.jp) for English language editing. This work was supported by JSPS KAKENHI Grant Number JP16K05019.

\section{Funding information}

The following funding is acknowledged: Japan Society for the Promotion of Science (grant No. JP16K05019).

\section{References}

Aoki, S., Ogata, T., Sudo, S. \& Onuki, T. (1992). Jpn. J. Appl. Phys. 31, 3477-3480.

Attwood, D. (1999). Soft X-rays and Extreme Ultraviolet Radiation, p. 361. Cambridge University Press.
Born, M. \& Wolf, E. (1986). Principles of Optics, 6th ed., p. 441. Oxford: Pergamon Press.

Erko, A., Agafonov, Yu., Panchenko, L. A., Yakshin, A., Chevallier, P., Dhez, P. \& Legrand, F. (1994). Opt. Commun. 106, 146-150.

Henke, B. L., Gullikson, E. M. \& Davis, J. C. (1993). At. Data Nucl. Data Tables, 54, 181-342.

Kagoshima, Y., Takano, H., Koyama, T., Tsusaka, Y. \& Saikubo, A. (2011). Jpn. J. Appl. Phys. 50, 022503.

Kagoshima, Y. \& Takayama, Y. (2018). Microsc. Microanal. 24(Suppl. S2), 280-281.

Kang, H. C., Stephenson, G. B., Liu, C., Conley, R., Macrander, A. T., Maser, J., Bajt, S. \& Chapman, H. N. (2005). Appl. Phys. Lett. 86, 151109.

Kang, H., Maser, J., Stephenson, G. B., Liu, C., Conley, R., Macrander, A. T. \& Vogt, S. (2006). Phys. Rev. Lett. 96, 127401.

Kirz, J. (1974). J. Opt. Soc. Am. 64, 301-309.

Koyama, T., Ichimaru, S., Tsuji, T., Takano, H., Kagoshima, Y., Ohchi, T. \& Takenaka, H. (2008). Appl. Phys. Expr. 1, 117003.

Koyama, T., Takano, H., Konishi, S., Tsuji, T., Takenaka, H., Ichimaru, S., Ohchi, T. \& Kagoshima, Y. (2012). Rev. Sci. Instrum. 83, 013705.

Li, K. \& Jacobsen, C. (2018). J. Synchrotron Rad. 25, 1048-1059.

Li, K., Wojcik, M. J., Divan, R., Ocola, L. E., Shi, B., Rosenmann, D. \& Jacobsen, C. (2017). J. Vac. Sci. Technol. B, 35, 06G901.

Maser, J. \& Schmahl, G. (1992). Opt. Commun. 89, 355-362.

Maser, J., Stephenson, G. B., Vogt, S., Yun, W., Macrander, A., Kang, H. C., Liu, C. \& Conley, R. (2004). Proc. SPIE, 5539, 185-195.

Michette, A. G. (1986). Optical Systems for Soft X-rays. New York: Plenum.

Mimura, H., Yumoto, H., Matsuyama, S., Sano, Y., Yamamura, K., Mori, Y., Yabashi, M., Nishino, Y., Tamasaku, K., Ishikawa, T. \& Yamauchi, K. (2007). Appl. Phys. Lett. 90, 051903.

Mohacsi, I., Vartiainen, I., Rösner, B., Guizar-Sicairos, M., Guzenko, V. A., McNulty, I., Winarski, R., Holt, M. V. \& David, C. (2017). Sci. Rep. 7, 43624.

Ozawa, A., Tamamura, T., Ishii, H., Yoshihara, H. \& Kagoshima, Y. (1997). Microelectron. Eng. 35, 525-529.

Rudolph, D., Niemann, B. \& Schmahl, G. (1982). Proc. SPIE, 0316, 103-105.

Schneider, G. (1997). Appl. Phys. Lett. 71, 2242-2244.

Schnopper, H. W., Van Speybroeck, L. P., Delvaille, J. P., Epstein, A., Källne, E., Bachrach, R. Z., Dijkstra, J. \& Lantward, L. (1977). Appl. Opt. 16, 1088-1091.

Schroer, C. G., Kurapova, O., Patommel, J., Boye, P., Feldkamp, J., Lengeler, B., Burghammer, M., Riekel, C., Vincze, L., van der Hart, A. \& Küchler, M. (2005). Appl. Phys. Lett. 87, 124103.

Snigireva, I., Snigirev, A., Kohn, V., Yunkin, V., Grigoriev, M., Kuznetsov, S., Vaughan, G. \& Di Michiel, M. (2007). Phys. Status Solidi A, 204, 2817-2823.

Takano, H., Tsuji, T., Hashimoto, T., Koyama, T., Tsusaka, Y. \& Kagoshima, Y. (2010). Appl. Phys. Expr. 3, 076702.

Wang, Y., Jacobsen, C., Maser, J. \& Osanna, A. (2000). J. Microsc. 197, 80-93. 